Apidologie, 1973, 4 (3), 267-274.

\title{
DIE ANZAHL DER SPERMIEN IM RECEPTACULUM SEMINIS VON HUMMELKÖNIGINNEN (HYM., APOIDEA, BOMBINAE)
}

\author{
Le nombre des spermatozoïdes dans le receptaculum seminis \\ des reines de Bourdons (Hym., Apoidea, Bombinae)
}

\author{
Peter-Frank RÖSELER ${ }^{1}$ \\ Zoologisches Institut II der Universität Würzburg
}

\begin{abstract}
SUMMARY
THE NUMBER OF SPERMATOZOA IN THE SPERMATHECA

of bumble bee queens (Hym., Apoidea, Bombinae)
\end{abstract}

The number of spermatozoa in the spermatheca of queens was evaluated for several species of bumble bees in order to test if, under natural conditions, single or multiple matings are occurring.

The amount of sperm was compared at :

1. queens which had been captured in autumn during mating time,

2. queens captured in spring,

3. queens with controlled single mating.

This comparison showed that, as a rule, queens are mated only once. Merely queens of Bombus hypnorum seem to be mated 2-3 times.

The males of Bombus are able to copulate repeatedly. During the first and second copulation the drones of $B$. hypnorum and $B$. terrestris discharged the same amount of sperm. At further copulations males of B. hypnorum discharge less spermatozoa than at the first and second time. The duration of copulation increases with the number of copulations. At the end of a colony's development the quantity of spermatozoa in the spermatheca of old queens does not differ significantly from that evaluated at the colony's start. Therefore, the quantity of sperm cannot be a limiting factor of the colony's strength.

Mit Unterstützung der Deutschen Forschungsgemeinschaft.

1. Anschrift : Dr. P.-F. Röseler, Zoologisches Institut II, D - 87 Würzburg, Rőntgenring 10. 


\section{ZUSAMMENFASSUNG}

Bei verschiedenen Hummelarten wurde die Anzahl der Spermien im Receptaculum seminis von Königinnen bestimmt, um zu prüfen, ob Königinnen in der freien Natur einmal oder mehrmals begattet werden. Verglichen wurden die Spermienmengen von :

1. Königinnen, die zur Paarungszeit im Herbst gefangen wurden,

2. Königinnen, die im Frühjahr gefangen wurden,

3. Königinnen, die kontrolliert nur lmal begattet waren.

Dieser Vergleich ergab, daß Königinnen in der Regel nur lmal begattet werden. Lediglich Königinnen von $B$. hypnorum scheinen auch 2-3mal begattet zu werden.

Hummeldrohnen können mehrmals kopulieren. Bei B. hypnorum und B. terrestris ist die Spermienmenge, die bei der 1 . und 2. Kopulation übertragen wird, gleich. Drohnen von $B$. hypnorum übertragen bei weiteren Kopulationen weniger Spermien als bei der I. und 2. Kopulation. Die Dauer der Kopulation nimmt mit der Anzahl der Kopulationen zu.

Die Anzahl der Spermien im Receptaculum seminis alter Königinnen am Ende der Volksentwicklung ist nicht signifikant verschieden von der Anzahl der Spermien zu Beginn der Volksentwicklung. Die Spermienmenge kann damit kein limitierender Faktor für die Volksstärke sein.

\section{I. - EINLEITUNG}

Eine erfolgreiche Hummelzucht setzt eine kontrollierte Begattung der Königinnen voraus. In früheren Untersuchungen (RösELER, 1967) hatte ich festgestellt, daß Königinnen mehrmals begattet wurden, wenn ich sie mit Drohnen zusammen in einem Gazekäfig hielt. Die Mehrzahl dieser Königinnen wurde 3-4mal begattet, einzelne Königinnen bis zu 9mal. Außerdem konnte ich beobachten, daß Drohnen mehrmals kopulierten. Ausgehend von diesen Beobachtungen untersuchte ich, ob eine Königin mehrmals begattet werden muß, um im Verlauf der Volksentwicklung eine ausreichende Menge besamter Eier legen zu können, oder ob eine einmalige Begattung ausreicht. Gleichzeitig untersuchte ich, ob Drohnen bei jeder Kopulation die gleiche Spermienmenge übertragen.

Das Kopulationsverhalten der Hummeln wurde bereits mehrfach beschrieben (Hoffer, 1882 /1883, Postner, 1953, Stein, 1956, Pouvreau, 1963 u.a.). Untersuchungen über die Anzahl der Spermien sind mir dagegen bisher nicht bekannt geworden.

\section{II. - METHODIK}

Die untersuchten Arten sind in den Tabellen aufgeführt. Die freifliegenden Königinnen wurden in der Umgebung von Freiburg und Würzburg gefangen. Königinnen und Drohnen für kontrollierte Begattungen entstammten meinen Zuchten. Die Tiere wurden individuell markiert und in kleine Gazekäfige zusammengesetzt.

Die Untersuchung der Spermien im Receptaculum seminis erfolgte 1 Tag nach der Begattung : Die Königinnen wurden abgetötet, das Receptaculum seminis herauspräpariert und in eine definierte Menge destillierten Wassers übertragen. Unter einem Stereomikroskop wurde 
das Receptaculum seminis aufpräpariert und die Spermien mit Hilfe von zwei Pinzetten gleichmäßig verteilt. Anschließend wurde ein Teil der Flüssigkeit in eine Zählkammer (Modell : FUCHS-ROSENTHAL) überführt und die Anzahl der Spermien unter dem Mikroskop bei etwa 200 facher Vergrößerung gezählt (Phasenkontrast). Im destillierten Wasser überleben die Spermien einige Minuten, wodurch sie zunächst leichter verteilt werden können und nicht verklumpen; in der Zählkammer bewegen sich die Spermien dann aber nicht mehr, so daß sie leicht gezählt werden können.

\section{III. - ERGEBNISSE}

Wenn Königinnen mehrmals begattet werden, müßte die Anzahl der Spermien einzelner Königinnen, die während der Paarungszeit im Herbst gefangen wurden, untereinander differieren, dagegen bei Königinnen, die im Frühjahr gefangen wurden, einheitlicher sein. Ich habe deshalb zunächst die Anzahl der Spermien von Königinnen im Herbst mit denen im Frühjahr verglichen. Die Ergebnisse zeigt die Tabelle 1.

Die Spermienmenge im Receptaculum seminis der Königinnen, die im Frühahr gefangen werden, kann als repräsentativ für die betreffenden Arten angesehen werden. Vergleicht man die einzelnen Arten, so fällt auf, daß die Anzahl der Spermien unterschiedlich ist. Die geringsten Spermienmengen habe ich bei Königinnen von $B$. pratorum (13 200) und B. variabilis (12 200) gefunden, die größten Spermienmengen bei $B$. terrestris (52 800).

Für einen statistischen Vergleich der Spermienmengen der Königinnen im Herbst mit den Spermienmengen der Königinnen im Frühjahr kann ich nur die Arten $B$. lapidarius, B. silvarum und $B$. terrestris heranziehen; denn nur diese Arten habe ich sowohl im Herbst wie im Frühjahr in ausreichender Anzahl gefangen. Der Vergleich bei diesen Arten zeigt, daß sich die Spermienmengen nicht signifikant unterscheiden. Aber auch die Einzelwerte der anderen Arten, die im Herbst gefangen wurden (B. agrorum, B. hortorum und B. pratorum), stimmen z.T. gut mit den Werten der Königinnen im Frühjahr überein.

Um zu sichern, daß die Königinnen in der Regel nur Imal begattet werden, habe ich die Werte von Herbst und Frühjahr verglichen mit der Spermienmenge, die bei 1 Begattung übertragen wird. Für die Arten B. lucorum und $B$. terrestris ergeben sich keine signifikanten Unterschiede. Von B. terrestris konnten auch Königinnen untersucht werden, die 2 mal begattet wurden. Die Anzahl der Spermien liegt im Mittel mit 77300 zwar höher als die Vergleichswerte, doch ist die Streuung sehr groß. Von $B$. hortorum wurde nur 1 Königin nach 1maliger Begattung untersucht, so daß nicht entschieden werden kann, ob es sich bei dem extrem niedrigen Wert um eine Ausnahme handelt, oder ob der Wert charakteristisch für eine lmalige Begattung ist. Die große Streuung der Werte bei $B$. variabilis entspricht derjenigen von Frühjahrsköniginnen.

Bei B. hypnorum ist die Anzahl der Spermien im Receptaculum seminis 


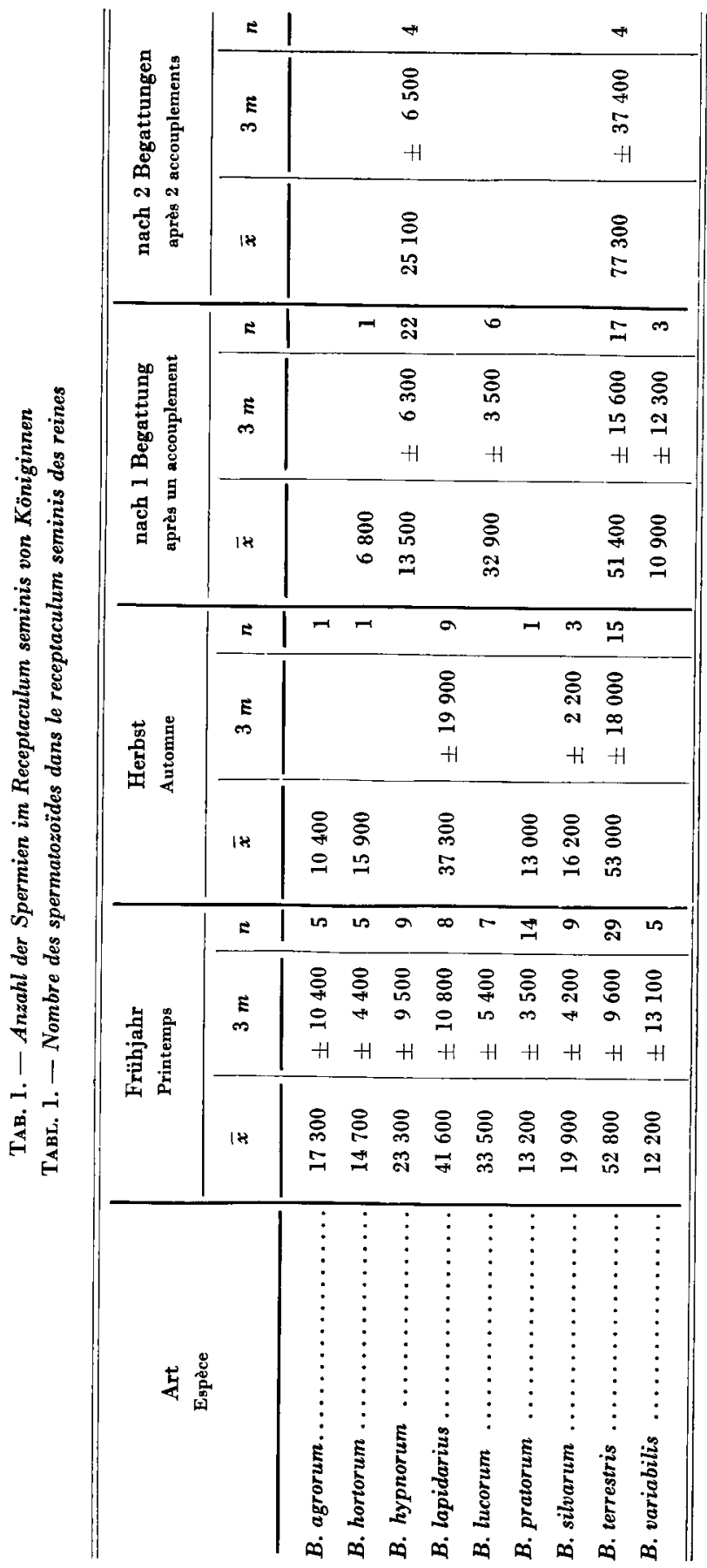


TAв. 2 - Anzahl der Spermien im Receptaculum seminis von B. hypnorum-Königinnen im Frühjahr TABL. 2. - Nombre des spermatozoïdes dans le receptaculum seminis de B. hypnorum au printemps

\begin{tabular}{|c|c|c|}
\hline $\begin{array}{l}\text { Anzahl der Spermien } \\
\text { Nombre des spermatozoides }\end{array}$ & $\begin{array}{l}\text { Mutmaßliche Häufigkeit } \\
\text { der Begattung } \\
\text { Nombre présumé d'accouplements }\end{array}$ & $\begin{array}{c}\text { Mutmaßliche Anzahl der } \\
\text { Spermien nach } 1 \text { Begattung } \\
\text { Nombre présumé des spermatozoides } \\
\text { après 1'accouplement }\end{array}$ \\
\hline 11000 & 1 & 11000 \\
\hline 12600 & 1 & 12600 \\
\hline 15400 & 1 & 15400 \\
\hline 22500 & 2 & 11200 \\
\hline 22800 & 2 & 11400 \\
\hline 24600 & 2 & 12300 \\
\hline 27900 & 2 & 13900 \\
\hline 33900 & 3 & 11300 \\
\hline 39300 & 3 & 13100 \\
\hline $\bar{x}=23300$ & & $\bar{x}=$ \\
\hline $3 m= \pm 9500$ & & $3 m= \pm 1600$ \\
\hline
\end{tabular}

von Königinnen im Frühjahr im Mittel $(=23300)$ fast doppelt so hoch wie die Anzahl nach 1 Begattung (= 13 500); der Unterschied zwischen beiden Gruppen ist signifikant $(P<1 \%)$. Die Spermienmenge der Königinnen im Frühjahr liegt in der Größenordnung der Spermienmenge der Königinnen, die 2 mal begattet wurden $(=25100)$. Ich vermutete daher, daß sich unter den im Frühjahr gefangenen Königinnen einige befanden, die mehrmals begattet worden waren, zumal auch die Streuung der Werte von Königinnen im Frühjahr größer ist als die der Vergleichswerte. Um dies zu prüfen, habe ich in Tabelle 2 die im Frühjahr gefundene Spermienmenge jeder einzelnen Königin durch die Anzahl der mutmaßlichen Begattungen dividiert. Dabei erhielt ich Werte zwischen 11000 und 14,000 Spermien pro Begattung. Diese Werte stimmen sehr gut mit den experimentell gefundenen Werten für 1 Begattung überein. Aus diesen Ergebnissen ziehe ich den Schluß, daß bei den B. hypnorum - Königinnen im Freien 1malige wie auch 2-3malige Begattung üblich ist.

Wie in der Einleitung erwähnt wurde, können Hummeldrohnen mehrmals kopulieren. Für eine kontrollierte Hummelzucht ist es deshalb notwendig zu wissen, ob nachfolgende Kopulationen auch erfolgreich sind. Ich habe deshalb bei $B$. hypnorum und B. terrestris die Anzahl der Spermien bestimmt, die von den Drohnen bei den einzelnen Kopulationen übertragen werden. Zugleich 


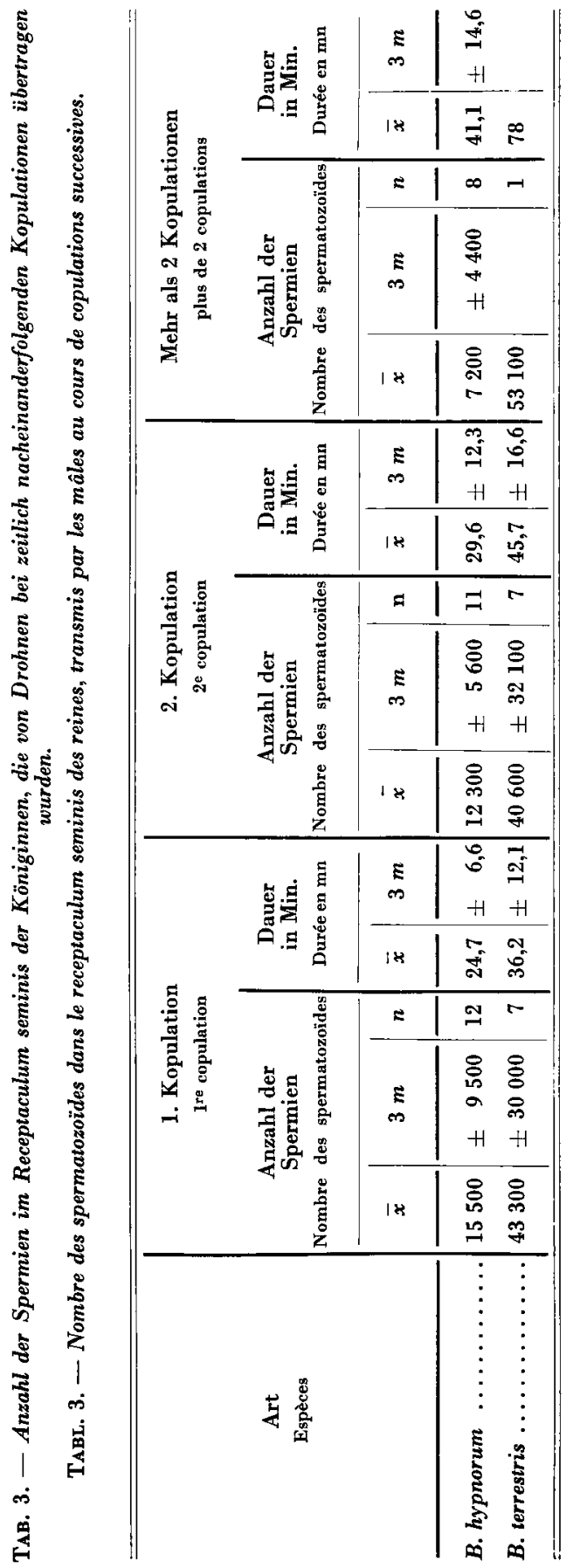


habe ich auch die Dauer der jeweiligen Kopulation registriert. Die Ergebnisse sind in der Tabelle 3 zusammengefaßt. Bei $B$. hypnorum haben einzelne Drohnen bis zu 5 mal kopuliert, doch habe ich diese Einzelwerte in eine Gruppe c mehr als 2 Kopulationen m zusammengefaßt.

Die Anzahl der Spermien, die von den Drohnen bei ihrer 1. und 2. Kopulation übertragen wurde, unterscheidet sich bei beiden Arten nicht. Dagegen nimmt bei B. hypnorum die Anzahl der Spermien bei weiteren Kopulationen ab $(P=5 \%)$. Von $B$. terrestris konnte nur einmal die Spermienmenge gezählt werden, die bei der 3. Kopulation übertragen wurde. Diese Anzahl entspricht dem Wert der beiden ersten Kopulationen.

Die Dauer der Kopulationen nimmt bei beiden Arten mit der Anzahl der Kopulationen zu. Bei B. hypnorum läßt sich der Wert bei « mehr als 2 Kopulationen ') gegenüber der 1 . Kopulation statistisch sichern $(\mathbf{P}<1 \%)$.

Da Drohnen mehrmals kopulieren können, wurde stichprobenartig untersucht, wieviele Spermien in den Hoden enthalten sind. Die Bestimmung ergab für B. lapidarius und B. terrestris jeweils etwa 750000 Spermien.

Vergleicht man die Anzahl der Spermien, die eine Königin zum Zeitpunkt der Volksgründung im Frühjahr besitzt, mit der Volksstärke, so kann man die Spermienmenge sicher nicht als limitierenden Faktor für die Volksentwicklung ansehen. Wenn man nämlich davon ausgeht, daß eine Königin von $B$. terrestris etwa 500 besamte Eier legt, dann dürfte sich dadurch die Anzahl der Spermien kaum verringern, auch wenn man Polyspermie annimmt. Um dies zu prüfen, habe ich einige alte Königinnen am Ende der Volksentwicklung untersucht. Die Ergebnisse bestätigen die obige Vermutung. Die Bestimmung ergab :
B. hypnorum
$14300 \pm 3500 \quad(n=4)$
B. terrestris
$39600 \pm 11700 \quad(n=7)$

Eingegangen im Februar 1973. Reçu pour publication en février 1973.

\section{RÉSUME}

Le nombre des spermatozoïdes présents dans le receptaculum seminis de reines de Bourdons appartenant à différentes espèces a été déterminé dans le but de savoir si, dans la nature, les reines sont fécondées une ou plusieurs fois. On a comparé les quantités de spermatozoïdes de :

1. reines capturées en automne à l'époque des accouplements,

2. reines capturées au printemps,

3. reines fécondées une seule fois dans des conditions contrôlées.

Cette comparaison a montré qu'en règle générale les reines ne sont fécondées qu'une seule fois. Seules quelques reines de $B$. hypnorum semblent avoir été fécondées 2 à 3 fois. 
Les mâles de bourdons peuvent copuler plusieurs fois. Chez B. hypnorum et B. terrestris la quantité de sperme qui est transmise est la même au cours de la $1^{\text {re }}$ et de la $2^{2}$ copulation. Les mâles de $B$. hypnorum transmettent moins de sperme au cours des copulations suivantes qu'au cours des $1^{\text {re }}$ et $2^{\mathrm{e}}$ copulations. La durée des copulations augmente avec le nombre des copulations.

Le nombre des spermatozoïdes présents dans le receptaculum seminis de vieilles reines à la fin de la période de développement des colonies n'est pas significativement différent du nombre des spermatozoïdes présents au début de ce développement. En conséquence, la quantité de spermatozoïdes présents ne peut pas constituer un facteur susceptible de limiter la force des colonies.

\section{LITERATURVERZEICHNIS}

Hoffer, E., 1882/83. Die Hummeln Steiermarks. Lebensgeschichte und Beschreibung derselben. Graz.

Postrer, M., 1953. Kopulationsverhalten bei den Gattungen Bombus und Psithyrus. Zool. Anz., 151, 300-306.

Pouvreau, A., 1963. Observations sur l'accouplement de Bombus hypnorum L. (Hym., Apidae) en serre. Ins. sociaux, 10, 111-118.

Röseler, P.-F., 1967. Die Entstehung, die Tätigkeiten und der Drüsenzustand der verschiedenen Formen im Hummelstaat (Untersuchungen an Bombush ypnorum (L.), Bombus terrestris (L.) u.a.). Dissertation, Freiburg.

StEIN, G., 1956. Beiträge zur Biologie der Hummel (B. terrestris L., B. lapidarius L. u.a.). Zool. Jahrb. (Morphologie), 84, 439-462. 